\title{
Prognostic significance of pseudocapsule status in patients with metastatic renal cell carcinoma treated with tyrosine kinase inhibitors
}

\author{
Wei Xi ${ }^{1 \#}$, Yingyong $\mathrm{Hou}^{2 \#}$, Xiaoyi Hu ${ }^{1 \#}$, Yu Xia ${ }^{1}$, Shuai Jiang ${ }^{1}$, Hang Wang ${ }^{1}$, Qi Bai ${ }^{1}$, Jun Hou ${ }^{2}$, \\ Jianming Guo
}

${ }^{1}$ Department of Urology, Zhongshan Hospital, Fudan University, Shanghai, China; ${ }^{2}$ Department of Pathology, Zhongshan Hospital, Fudan University, Shanghai, China

Contributions: (I) Conception and design: J Guo, W Xi; (II) Administrative support: J Guo; (III) Provision of study materials or patients: Y Hou, X Hu; (IV) Collection and assembly of data: X Hu, Y Xia, S Jiang; (V) Data analysis and interpretation: W Xi, Y Hou, X Hu, H Wang, Q Bai, J Hou; (VI) Manuscript writing: All authors; (VII) Final approval of manuscript: All authors.

\#These authors contributed equally to this work.

Correspondence to: Jianming Guo. Department of Urology, Zhongshan Hospital, Fudan University, Shanghai 200032, China.

Email: guo.jianming@zs-hospital.sh.cn.

\begin{abstract}
Background: We sought to determine whether pseudocapsule (PS) features have prognostic implications in patients with metastatic renal cell carcinoma (mRCC).

Methods: We retrospectively reviewed 231 patients diagnosed with mRCC and treated with tyrosine kinase inhibitors; 188 patients with data available regarding the tumor-parenchyma interfacial PS of the primary tumor were enrolled for analysis. PS status was evaluated as intact (grade 0), merely involved (grade 1), penetrated (grade 2), and absent (grade 3). We applied the Kaplan-Meier method and Cox regression model to assess the survival impact.

Results: Of the 188 patients, 19 (10.1\%), 61 (32.4\%), 96 (51.1\%) and 12 (6.4\%) had grade 0, 1, 2 and 3 PS, respectively. PS status was significantly associated with histology $(\mathrm{P}=0.0206)$, venous tumor embolus $(\mathrm{P}=0.0511)$, microvascular invasion $(\mathrm{P}=0.0108)$ and microsatellite formation $(\mathrm{P}=0.0097)$. Patients without a PS had the worst overall survival (OS), with a 3 -year OS rate of $12.7 \%$, whereas the OS rates for grades 0 , 1 and 2 were $78.8 \%, 50.8 \%$ and 43.6\%, respectively. Adjusted by other variables, grade 3 and grade 2 PS gave rise to a much higher risk of death across the cohort [hazard ratio $(\mathrm{HR})=5.217, \mathrm{P}=0.0182 ; \mathrm{HR}=3.765$, $\mathrm{P}=0.0281$, respectively]. Sarcomatoid change was also an independent factor for $\mathrm{OS}$ ( $\mathrm{HR}=2.932, \mathrm{P}=0.0075)$. In contrast, microsatellite formation was not associated with survival in the cohort.

Conclusions: PS status has prognostic implications for OS in metastatic renal cancer. The absence of the PS and sarcomatoid change are two pathological features related to an extremely poor prognosis.
\end{abstract}

Keywords: Metastatic renal cell carcinoma (mRCC); tumor-parenchyma interface (TPI); pseudocapsule (PS); prognosis; tyrosine kinase inhibitors (TKIs)

Submitted May 12, 2021. Accepted for publication Sep 22, 2021.

doi: $10.21037 /$ tau-21-429

View this article at: https://dx.doi.org/10.21037/tau-21-429

\section{Introduction}

Metastatic renal cell carcinoma (mRCC) has a dismal but heterogeneous prognosis (1). Laboratory indicators have been identified to greatly impact prognosis. Mainly based on these indicators, several prognostic models were developed in succession [e.g., Memorial Sloan Kettering Cancer Center (MSKCC), International Metastatic RCC Database Consortium (IMDC)], but recent models have not shown 
much improvement in predictive accuracy, indicating that a ceiling has been reached $(2,3)$. In the literature, traditional clinicopathologic factors of the primary tumor, such as pT stage, Fuhrman grade and sarcomatoid change, have been consistently reported to have an impact on clinical outcome even after metastasis (4-6). However, pathological parameters at the tumor-parenchyma interface (TPI) have not been well studied.

The pseudocapsule (PS) of the TPI is a common phenomenon in malignancies (7-9). An infiltrative pattern of the PS has been frequently observed. In kidney cancer, 10-33\% patients were reported to have a penetrated PS, and this rate is much higher than renal capsule (10-14). In addition, the extent of PS invasion could reflect the burden of cancer cells entering the circulation, and venous tumor thrombus (VTT) and microvascular invasion (MVI) are remarkably more frequent when the PS is penetrated or absent (15). Consequently, PS penetration and absence indicate a significantly increased risk of death and progression $(15,16)$. In recent years, a study reported the pathological impact of axitinib therapy and found a significantly strengthened PS at the TPI, suggesting plasticity of the anatomic structure (17). However, studies of PS status in mRCC patients remain rare. The hypothesis of the study was that the PS at the TPI has prognostic implications in mRCC. We sought to assess this hypothesis by analyzing a cohort of mRCC patients treated with tyrosine kinase inhibitors (TKIs). We present the following article in accordance with the REMARK reporting checklist (available at https://dx.doi. org/10.21037/tau-21-429).

\section{Methods}

The retrospective cohort study was carried out at Zhongshan Hospital, Fudan University. With archived data from our institution, the inclusion criteria were as follows: patients received kidney surgery (partial, radical or cytoreductive nephrectomy) between 2004 and 2017 at our institution and were pathologically diagnosed with renal cell carcinoma (RCC); M1 stage was reached at or after surgery; patients received sunitinib or sorafenib as first-line therapy; no history of other malignancies. The exclusion criteria were as follows: inadequate clinical or pathological data or unevaluable PS. Finally, 188 out of 231 cases were included in the analyses. Second-line treatment included other TKIs, cytokine therapy, traditional Chinese medicine and supportive treatment. No programmed cell death protein 1 (PD-1)/programmed death ligand 1 (PD-L1) inhibitors were used. The study was conducted in accordance with the Declaration of Helsinki (as revised in 2013). The study was approved by Zhongshan Hospital Clinical Research Ethics Committee (No. B2016-158) and individual consent for this retrospective analysis was waived.

We collected clinicopathological data from archived records. Tumors were staged according to the 2010 American Joint Committee on Cancer (AJCC) TNM classification. Tumor Fuhrman grade was determined according to the World Health Organisation (WHO)/ International Society of Urologic Pathologists (ISUP) 2016 criteria. The PS status of primary tumors was classified into four categories as we previously reported (15): grade 0 for intact PS, grade 1 for involved but not penetrated PS, grade 2 for penetrated PS with renal parenchyma involvement, and grade 3 for PS absence (Figure 1). To ensure that it was PS being assessed (rather than the renal capsule or perirenal capsule), assessment was only carried out when the parenchyma was observed on the other side.

The main endpoints were overall survival (OS) and progression-free survival (PFS), calculated from TKI treatment initiation. All patients underwent computed tomography scans to evaluate the response to TKI treatment according to the Revised Response Evaluation Criteria in Solid Tumors (RECIST) guidelines (version 1.1) (18).

\section{Statistical analysis}

The chi-square test and ANOVA were used to evaluate the associations among variables. The Kaplan-Meier method and log-rank test were applied to determine the survival difference. The hazard ratio (HR) and corresponding 95\% confidence interval (CI) were calculated with a Cox proportional model. $\mathrm{P}$ less than 0.05 was considered to be significant. Analyses were done with the $\mathrm{R}$ environment (version 3.3.2).

\section{Results}

\section{Basic clinical characteristics}

The characteristics of the 188 patients are summarized in Table 1. Clear cell, papillary and chromophobe cases accounted for $86.2 \%, 7.4 \%$ and $6.4 \%$, respectively. As many as $39.9 \%$ of tumors were initially localized at diagnosis but progressed to M1 after surgery; 29.3\% were simultaneously metastatic at surgery. According to the IMDC criteria, $18.6 \%, 65.4 \%$ and $16.0 \%$ of patients were classified into 

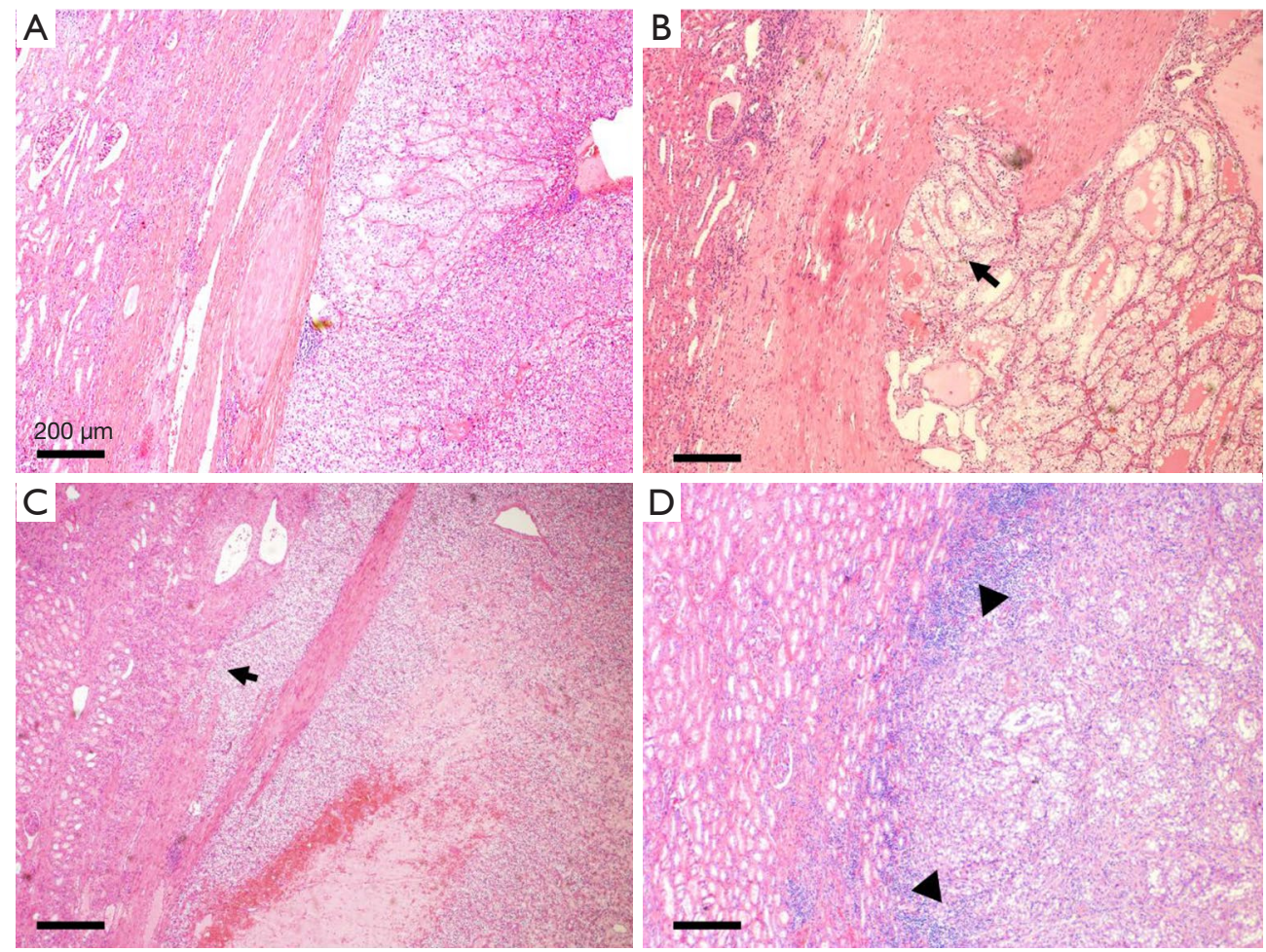

Figure 1 Representative pictures of PS status. (A) Grade 0 for intact PS (hematoxylin-eosin, original magnification $\times 40$ ); (B) grade 1 for merely involved but not penetrated PS (hematoxylin-eosin, original magnification $\times 40$ ); (C) grade 2 for penetrated PS with renal parenchyma involved (hematoxylin-eosin, original magnification $\times 40$ ); (D) grade 3 for PS absence (hematoxylin-eosin, original magnification $\times 40)$. Black arrows indicate where cancer invades or penetrates PS. Enhanced inflammation could be observed in (D) as indicated with black arrowheads. PS, pseudocapsule.

the favorable, intermediate and unfavorable risk groups, respectively. Fifty-nine-point-six percent and $40.4 \%$ of the patients were treated with sunitinib and sorafenib, respectively. The median follow-up time was 30.9 months after TKI therapy initiation.

\section{Associations with clinicopathological parameters}

There is a high rate of PS involvement in mRCC. In total, 19 (10.1\%), 61 (32.4\%), $96(51.1 \%)$ and 12 (6.4\%) tumors had grade 0, 1, 2 and 3 PS status, respectively. Compared to those with clear cell subtype, patients with non-clear cell histology had a lower rate of PS absence (4.9\% vs. $15.4 \%$, $\mathrm{P}=0.0206)$. The rates of MVI significantly increased with PS grade $(\mathrm{P}=0.0108)$ and tumor embolus increased had the similar association with marginal significance $(\mathrm{P}=0.0511)$. The MVI and embolus rates in tumors with grade $2 / 3$ PS were $83 \%$ and $73 \%$, respectively. PS was not associated with nuclear grade $(\mathrm{P}=0.0975)$, initial stage $(\mathrm{P}=0.3202)$ or IMDC risk stratification $(\mathrm{P}=0.3132)$. In addition, $20.2 \%$ of tumors had at least one site of microsatellite formation, which was associated with higher PS grade (grade 2 and 3, P=0.0097) (Table 1).

\section{Prognostic value of PS status}

During follow-up, a total of 118 patients (62.8\%) experienced progression and $96(51.1 \%)$ died. PS status was not associated with PFS (Figure $2 A, \mathrm{P}=0.376$ ) or therapy response $(\mathrm{P}=0.596$, Table $\mathrm{S} 1)$, but it stratified $\mathrm{OS}$ well (Figure $2 B, \mathrm{P}=0.0117$ ). The 1.5 -year and 3 -year survival rates of patients with grade 3 PS were the lowest $38.1 \%$ and $12.7 \%)$ compared to those with grade $0(78.8 \%$ and $78.8 \%)$, grade $1(70.9 \%$ and $50.8 \%)$ and grade 2 PS $(61.0 \%$ and $43.6 \%)$. Microsatellite formation was not associated with survival $(\mathrm{P}=0.6120)$. Sarcomatoid change resulted in an increased risk of death with marginal significance $(\mathrm{P}=0.0632)$. IMDC, MVI, nuclear grade and lung metastasis were also 
Table 1 Basic characteristics and relations of PS status with other variables

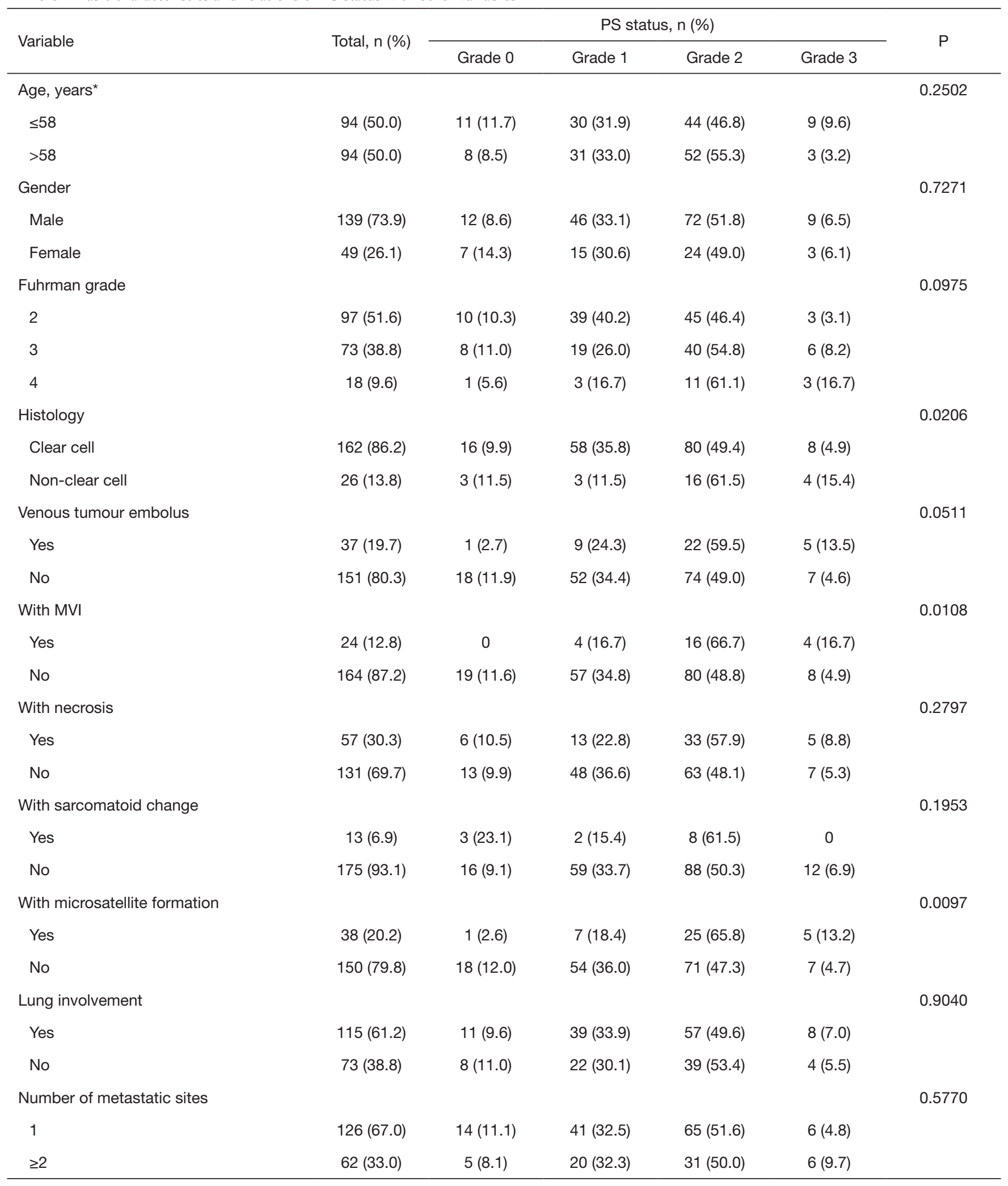

Table 1 (continued) 
Table 1 (continued)

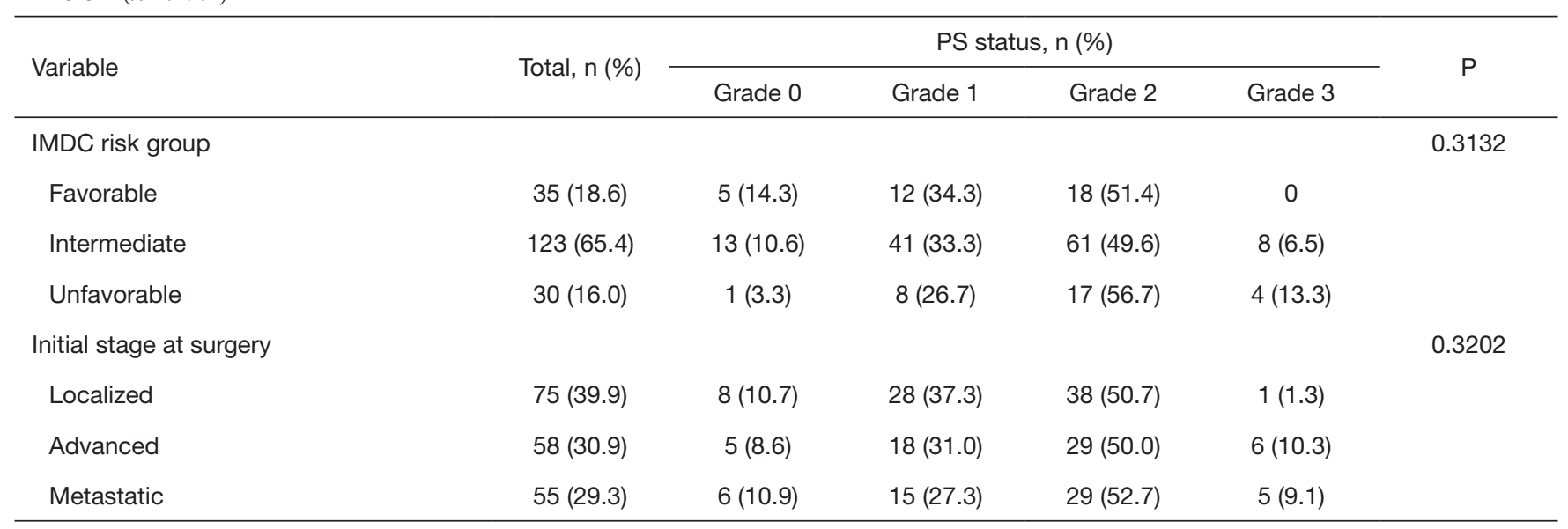

*, split at median value. PS, pseudocapsule; MVI, microvascular invasion; IMDC, International Metastatic Renal Cancer Database Consortium.

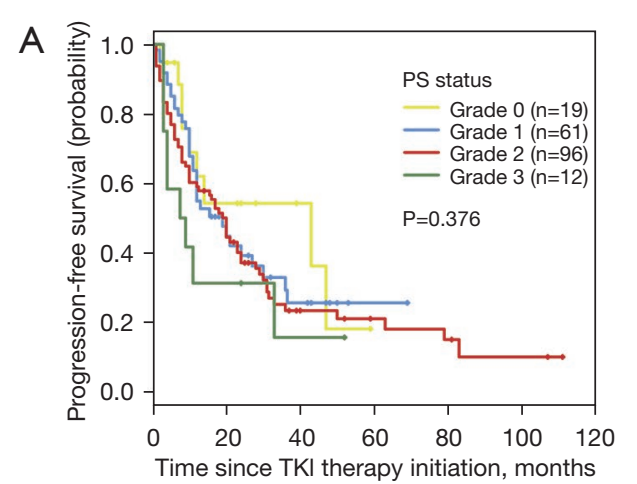

$\begin{array}{lcccccc}\text { Number at risk } & & & & \\ \text { Grade 0 } & 19 & 7 & 3 & 0 & 0 & 0 \\ \text { Grade 1 } & 61 & 17 & 7 & 1 & 0 & 0 \\ \text { Grade 2 } & 96 & 36 & 11 & 6 & 4 & 2 \\ \text { Grade 3 } & 12 & 3 & 1 & 0 & 0 & 0\end{array}$

B

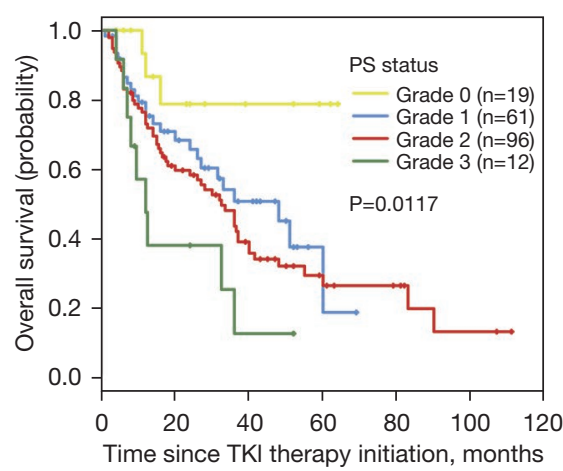

Number at risk

$\begin{array}{ccccccc}\text { Grade 0 } & 19 & 11 & 7 & 2 & 0 & 0 \\ \text { Grade 1 } & 61 & 28 & 15 & 2 & 0 & 0 \\ \text { Grade 2 } & 96 & 47 & 24 & 10 & 6 & 2 \\ \text { Grade 3 } & 12 & 4 & 1 & 0 & 0 & 0\end{array}$

Figure 2 Kaplan-Meier curves of survival according to time after therapy initiation and PS status in mRCC. (A) PFS; (B) OS. mRCC, metastatic renal cell carcinoma; PS, pseudocapsule; PFS, progression-free survival; OS, overall survival; TKI, tyrosine kinase inhibitor.

significant in the univariate analysis (Table 2). In multivariate analysis, PS status and sarcomatoid change persisted to be two pathological prognostic parameters (Table 3). The absence and penetration of PS resulted in a 4.2- and 2.7-fold higher risk of death to grade $0 \mathrm{PS}$, respectively $(\mathrm{HR}=5.217$, $\mathrm{P}=0.0182 ; \mathrm{HR}=3.765, \mathrm{P}=0.0281$ ). The survival benefit of grade 1 to 0 was marginal $(\mathrm{P}=0.0524)$. Nuclear grade lost the influence on OS in multivariate analysis.

Although PS absence and sarcomatoid change were only observed in $13.3 \%$ of all cases $(n=25)$, they were related to remarkably poor survival. Patients with one of the features made those in favorable or intermediate risk group have clinical outcomes as poor as those in the unfavorable risk group (Figure S1). Integrating PS status into the IMDC model increased the C-index for survival from 0.64 to 0.73 across the cohort.

\section{Discussion}

There are few studies on PS status in renal cancer and even fewer studies in mRCC. The present study demonstrated that PS status in mRCC was far different from that in 
Table 2 Univariable analyses for OS

\begin{tabular}{|c|c|c|c|}
\hline Variable & $\mathrm{HR}$ & $95 \% \mathrm{Cl}$ & $P$ \\
\hline Gender (female vs. male) & 0.889 & $0.556-1.423$ & 0.6250 \\
\hline Histology (non-clear cell vs. clear cell) & 1.358 & $0.793-2.325$ & 0.2660 \\
\hline MVI (with vs. without) & 1.746 & $1.005-3.037$ & 0.0480 \\
\hline Sarcomatoid change (with vs. without) & 2.004 & $0.962-4.173$ & 0.0632 \\
\hline Fuhrman grade (3+4 vs. 2) & 1.582 & $1.057-2.368$ & 0.0259 \\
\hline Systematic therapy (sunitinib vs. sorafenib) & 0.9454 & $0.615-1.454$ & 0.7980 \\
\hline Lung involvement (with vs. without) & 1.797 & $1.159-2.787$ & 0.0087 \\
\hline Number of involved organs ( $\geq 2$ vs. 1 ) & 1.213 & $0.796-1.847$ & 0.3690 \\
\hline \multicolumn{4}{|l|}{ Initial stage at surgery } \\
\hline Localized & Reference & & \\
\hline Advanced & 1.223 & $0.744-2.008$ & 0.4271 \\
\hline Metastatic & 1.519 & $0.934-2.468$ & 0.0917 \\
\hline \multicolumn{4}{|l|}{ IMDC risk group } \\
\hline Favorable & Reference & & \\
\hline Intermediate & 1.717 & $0.900-3.276$ & 0.1008 \\
\hline Lack of PS & 7.130 & $1.924-26.420$ & 0.0032 \\
\hline Microsatellite formation (with vs. without) & 1.139 & $0.688-1.885$ & 0.6120 \\
\hline
\end{tabular}

$\mathrm{Cl}$, confidence interval; HR, hazard ratio; IMDC, International Metastatic Renal Cancer Database Consortium.; MVI, microvascular invasion; MVI, microvascular invasion; OS, overall survival; PS, pseudocapsule.

patients without metastasis and was significantly associated with survival. Although PS absence was only observed in a small proportion of samples, it resulted in remarkable survival loss. Thus, the treatment and monitoring of patients with unfavorable clinicopathological features (PS absence or sarcomatoid change) should follow a stricter approach.

The PS is the fibrous interface between the tumor and renal parenchyma. It has been found to be frequently invaded and penetrated by cancer cells. In early stage of RCC (p/cT1-2), the penetration ratio reaches $10-33 \%$ (10-14). In a large cohort from our institution, $20 \%$ of nonmetastatic tumors and $18 \%$ of localized tumors penetrated the PS. The proportion reached nearly $50 \%$ in mRCC in the present cohort (15). Of note, all assessments were based on pathology specimens after surgery, indicating that the extent of potential aggressiveness could be revealed as early as the time of surgery. This could also be 
Table 3 Multivariable analyses for OS

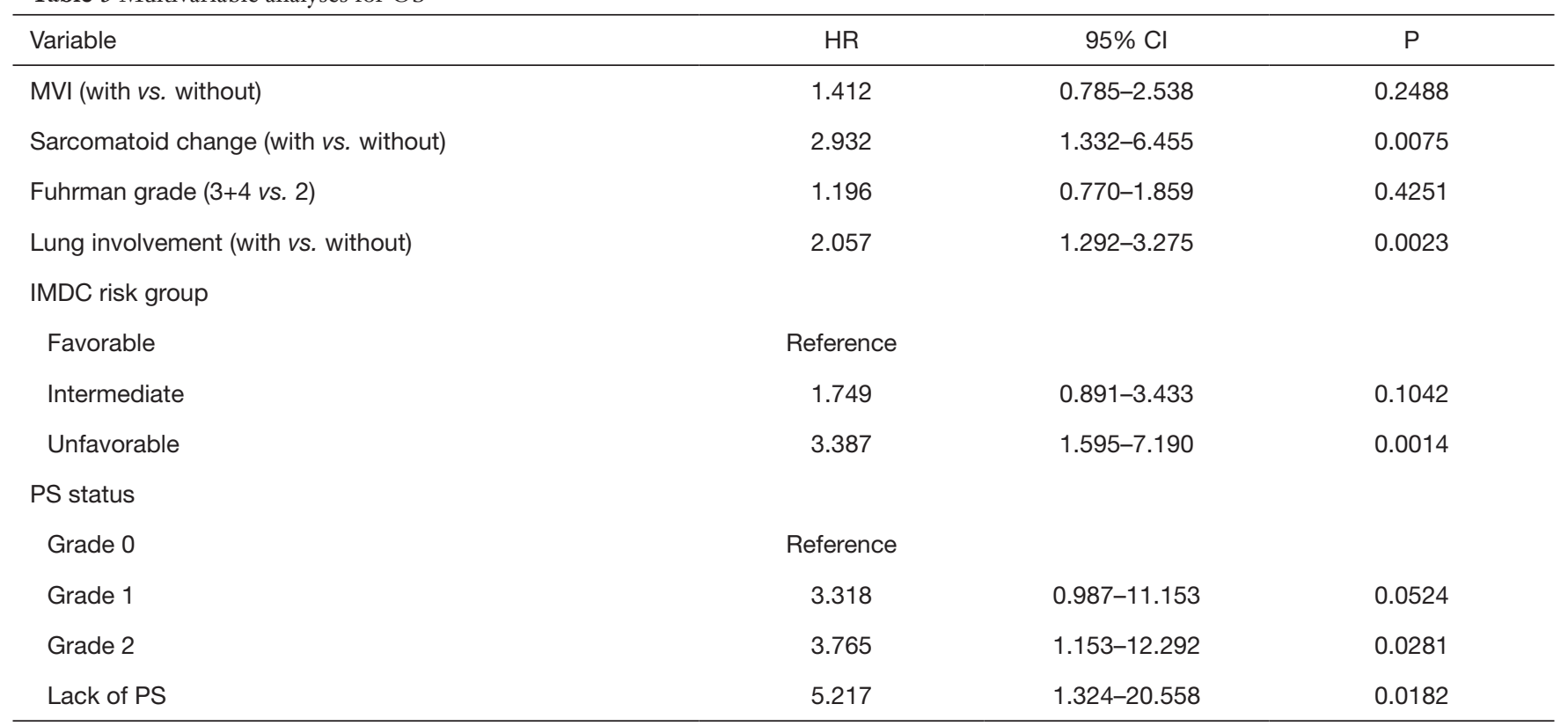

$\mathrm{Cl}$, confidence interval; HR, hazard ratio; IMDC, International Metastatic Renal Cancer Database Consortium.; MVI, microvascular invasion; MVI, microvascular invasion; OS, overall survival; PS, pseudocapsule.

complemented by the comparable distribution of PS status in different initial stage groups $(\mathrm{P}=0.32)$. PS penetration in different initial stage groups was the same $(\sim 50 \%)$. The only exception was that patients with a higher initial stage had higher rates of PS absence (initial nonlocalized $v s$. localized: $9.7 \%$ vs. $1.3 \%, \mathrm{P}=0.04)$.

Although the prognostic assessment of mRCC mostly depends on laboratory indicators, anatomic or traditional factors of the primary lesion could be useful. Kim et al. (4) found that initial $\mathrm{pT}$ stage is an independent factor for cancer-specific survival in mRCC. Its prognostic value was stronger than that of any molecular marker discussed in the report [carbonic anhydrase 9 (CA9), phosphatase and tensin homolog (PTEN), p53, vimentin]. Combining clinical and molecular markers achieved the best predictive accuracy. In another study, Tosco et al. (5) investigated the role of initial clinical factors in mRCC patients who underwent nephrectomy and at least one metastasectomy. He proved that $\mathrm{T}$ stage $\geq 3$ and Fuhrman grade $\geq 3$ separately led to a 2.3-2.8 higher risk of cancer-specific death. A model purely based on clinical factors (including $\mathrm{T}$ stage and Fuhrman grade) was efficient [area under the curve (AUC) at 5-year $=0.88]$. In contrast, Leibovich et al. (6) did not detect any influential role of $\mathrm{T}$ stage or Fuhrman grade but revealed a good predictive role of sarcomatoid change, which was also verified by other reports (19). In the present study, we did not find prognostic capability of initial TNM stage. Survival decreased with increasing Fuhrman grade, but it was nonsignificant in multivariate analysis. Only PS status and sarcomatoid change were pathological factors of prognostic value.

The prognostic significance of PS status was also reported in the liver metastases of gastrointestinal cancer. Patients with a thin PS or without a PS had significantly decreased survival (20). Here, we provided the first evidence of the relationship between PS status and oncological outcome in mRCC. Specifically, patients without a PS experienced extremely poor survival, with a median survival time of only 10.7 months. Notably, the PS of the primary tumor rather than metastatic lesion was assessed because metastases from RCC had nearly no PS (figure not shown).

PS is associated not only with prognosis but also with plasticity with treatment. In a cohort of 74 patients with soft tissue sarcoma, O'Donnell et al. (21) found that neoadjuvant chemotherapy before surgery contributed to the development of a PS. A similar finding was also reported for the liver metastasis of colorectal cancer (22). In kidney cancer, a recent study with 46 clear cell RCC patients discussed pathological changes at TPI after axitinib treatment (17). Compared with the control group 
$(\mathrm{n}=23$, surgery alone), the group with neoadjuvant axitinib treatment (5 $\mathrm{mg}$ for up to 12 weeks) had significantly higher PS grade, whereas the degree of glomerulosclerosis, arteriosclerosis and nephrosclerosis remained unchanged $(n=23)$. This phenomenon indicated that the PS could be reconstructed in a short period of time. We wondered whether PS was associated with the TKI response, which was denied by the results (Table S1). Generally, the current efficient models or indicators are more related to OS (2). In this study, the phenomenon also applies to PS status.

Three percent of RCC cases are observed to have sarcomatoid change. The presence of this feature may be one of the most influential prognostic variables for clinical outcome (19). The majority of series report a median survival of 4-9 months (23-25). The median survival in the present study was 4 months. We verified that patients without a PS also had rather dismal outcomes. The two features could help to distinguish a small group of patients with high risk whatever the status of other indicators. Meanwhile, we also found that patients with sarcomatoid change all had a PS at TPI, which was contradictory to the intuition. Given the limited cases, more studies are needed to verify our findings.

The PS could be an important means by which cancer cells spread. We previously found that the incidence of venous embolus and MVI, direct evidence of cancer cells entering the circulation, rises dramatically with increasing PS grade (15). The phenomenon was also found in this study. A total of $18.5 \%$ of patients with grade $2 / 3$ PS had MVI, whereas the rate was only $5 \%$ in the grade $0 / 1$ group. The association with tumor venous embolus was similar (Table 1). Invasive tumors could have a small subgroup of cancer cells that can collectively invade new regions through cell-cell and cell-matrix interactions, thus facilitating metastasis (26). In several other non-metastatic clear cell RCC cases, we observed that the PS area and the close peri-PS area contained the most abundant veins with large diameters (long diameter $>200 \mu \mathrm{m}$ ). They went diagonally through the PS, which further increased the size of the tumor-facing surface. In many situations, the leading-edge cancer cells were only separated by a thin layer of endotheliocytes from large veins (Figure S2). CD44, the indicator of invasion ability, was mainly distributed along the boundary between PS and tumors. They were further enriched surrounding large vessels (Figure S3, another case). Therefore, cancer cells near the PS are more aggressive, and the vasculature of the PS potentially provides a pathway for cancer cells to escape the primary site. Targeting these vessels could have therapeutic effects. In fact, Kawakami et al.'s report to some degree verified that these vessels of PS are undeniable targets during axitinib therapy (17). But initial PS status was not associated with TKI response or PFS, but significantly associated with OS, indicating that treatment response is complicated which might be determined by many unknown factors.

Microsatellite formation was also discussed in malignancies. In a study involving 278 hepatocellular carcinoma (HCC) patients (27), both PS and microsatellite formation had a survival impact, although the latter lost significance in multivariate analysis. Patients with microsatellite formation had a median disease-free survival (DFS) time of only 6.3 months, whereas other patients had a median DFS time of 14.9 months $(\mathrm{P}=0.0006)$. Similar findings were observed in two other studies in HCC $(20,28)$. In the present study, we detected a close relationship between PS status and microsatellite formation in renal cancer, but the latter did not effectively stratify patients according to prognosis $(\mathrm{P}=0.6120)$.

Metastatic site and number of metastatic lesions could impact survival greatly. Motzer et al. found that liver metastasis and increased metastatic number indicated deteriorated outcomes (29). In another report enrolled 113 mRCC patients with the intermediate risk group, bone and brain metastasis had a negative effect on OS (30). However, we in the present study found that patients with lung metastasis on the contrary experienced worse survival than those with other metastatic sites. This could be influenced by the factor that in our study patients with lung metastasis had more metastatic sites, indicating increased tumor burden in these patients. The finding was previously reported with a smaller cohort from our institution (31), suggesting a stable phenomenon.

The main limitations of this study are its retrospective nature and relatively small sample size. All assessments relied on preserved slides, and the exclusion criteria could lead to potential selection bias. Although strict followup made the cohort typical, the findings in the study still need to be verified by other institutions with a large cohort, especially with more cases without a PS. In addition, we did not include patients treated with other TKIs (such as pazopanib) because they were unavailable at that time. Given the huge survival difference among patients treated with different TKIs, the prognostic value of PS status in these patients needs to be further studied.

In conclusion, we found that PS status has prognostic implications for OS in metastatic renal cancer. The absence 
of the PS and sarcomatoid change are two pathological features related to an extremely poor prognosis.

\section{Acknowledgments}

Funding: This study was supported by Shanghai Sailing Program (19YF1408000) and Shanghai Anticancer Association EYAS PROJECT (SACA-CY1B11). All these study sponsors have no roles in the study design, collection, analysis, and interpretation of data.

\section{Footnote}

Reporting Checklist: The authors have completed the REMARK reporting checklist. Available at https://dx.doi. org/10.21037/tau-21-429

Data Sharing Statement: Available at https://dx.doi. org/10.21037/tau-21-429

Conflicts of Interest: All authors have completed the ICMJE uniform disclosure form (available at https://dx.doi. org/10.21037/tau-21-429). The authors have no conflicts of interest to declare.

Ethical Statement: The authors are accountable for all aspects of the work in ensuring that questions related to the accuracy or integrity of any part of the work are appropriately investigated and resolved. The study was conducted in accordance with the Declaration of Helsinki (as revised in 2013). The study was approved by Zhongshan Hospital Clinical Research Ethics Committee (No. B2016158) and individual consent for this retrospective analysis was waived.

Open Access Statement: This is an Open Access article distributed in accordance with the Creative Commons Attribution-NonCommercial-NoDerivs 4.0 International License (CC BY-NC-ND 4.0), which permits the noncommercial replication and distribution of the article with the strict proviso that no changes or edits are made and the original work is properly cited (including links to both the formal publication through the relevant DOI and the license). See: https://creativecommons.org/licenses/by-nc-nd/4.0/.

\section{References}

1. Cohen HT, McGovern FJ. Renal-cell carcinoma. N Engl J
Med 2005;353:2477-90.

2. Heng DY, Xie W, Regan MM, et al. Prognostic factors for overall survival in patients with metastatic renal cell carcinoma treated with vascular endothelial growth factortargeted agents: results from a large, multicenter study. J Clin Oncol 2009;27:5794-9.

3. Motzer RJ, Bacik J, Murphy BA, et al. Interferon-alfa as a comparative treatment for clinical trials of new therapies against advanced renal cell carcinoma. J Clin Oncol 2002;20:289-96.

4. Kim HL, Seligson D, Liu X, et al. Using tumor markers to predict the survival of patients with metastatic renal cell carcinoma. J Urol 2005;173:1496-501.

5. Tosco L, Van Poppel H, Frea B, et al. Survival and impact of clinical prognostic factors in surgically treated metastatic renal cell carcinoma. Eur Urol 2013;63:646-52.

6. Leibovich BC, Cheville JC, Lohse CM, et al. A scoring algorithm to predict survival for patients with metastatic clear cell renal cell carcinoma: a stratification tool for prospective clinical trials. J Urol 2005;174:1759-63; discussion 1763.

7. Verhagen JN, Van der Heijden MC, Rijksen G, et al. Determination and characterization of hexokinase in thyroid cancer and benign neoplasms. Cancer 1985;55:1519-24.

8. Lu Q, Ji C, Zhao X, et al. Histopathologic analysis of tumor bed and peritumoral pseudocapsule after in vitro tumor enucleation on radical nephrectomy specimen for clinical T1b renal cell carcinoma. Urol Oncol 2017;35:603. e15-20.

9. Okano K, Yamamoto J, Kosuge T, et al. Fibrous pseudocapsule of metastatic liver tumors from colorectal carcinoma. Clinicopathologic study of 152 first resection cases. Cancer 2000;89:267-75.

10. Minervini A, Serni S, Di Cristofano C, et al. Rebuttal from authors re: Vincenzo Ficarra, Antonio Galfano and Stefano Cavalleri. Is simple enucleation a minimal partial nephrectomy responding to the EAU guidelines' recommendations? Eur Urol 2009;55:1315-8. Eur Urol 2009;55:1319-20.

11. Cho HJ, Kim SJ, Ha US, et al. Prognostic value of capsular invasion for localized clear-cell renal cell carcinoma. Eur Urol 2009;56:1006-12.

12. Jeong IG, Jeong CW, Hong SK, et al. Prognostic implication of capsular invasion without perinephric fat infiltration in localized renal cell carcinoma. Urology 2006;67:709-12.

13. Klatte T, Chung J, Leppert JT, et al. Prognostic relevance 
of capsular involvement and collecting system invasion in stage I and II renal cell carcinoma. BJU Int 2007;99:821-4.

14. May M, Brookman-Amissah S, Roigas J, et al. Evaluation of renicapsular involvement in Stages I and II renal cell carcinoma from the morphological and prognostic point of view. Urol Oncol 2010;28:274-9.

15. Xi W, Wang J, Liu L, et al. Evaluation of Tumor Pseudocapsule Status and its Prognostic Significance in Renal Cell Carcinoma. J Urol 2018;199:915-20.

16. Volpe A, Bollito E, Bozzola C, et al. Classification of Histologic Patterns of Pseudocapsular Invasion in OrganConfined Renal Cell Carcinoma. Clin Genitourin Cancer 2016;14:69-75.

17. Kawakami F, Rao P, Tamboli P, et al. Study of the Kidney Tumor-Parenchymal Interface after Neoadjuvant Treatment with Axitinib for Locally Advanced Clear Cell Renal Cell Carcinoma: Matched Analysis from a Phase II Trial. J Urol 2017;197:559-65.

18. Eisenhauer EA, Therasse P, Bogaerts J, et al. New response evaluation criteria in solid tumours: revised RECIST guideline (version 1.1). Eur J Cancer 2009;45:228-47.

19. Shuch B, Bratslavsky G, Linehan WM, et al. Sarcomatoid renal cell carcinoma: a comprehensive review of the biology and current treatment strategies. Oncologist 2012;17:46-54.

20. Kemeny F, Vadrot J, Wu A, et al. Morphological and histological features of resected hepatocellular carcinoma in cirrhotic patients in the West. Hepatology 1989;9:253-7.

21. O'Donnell PW, Manivel JC, Cheng EY, et al. Chemotherapy influences the pseudocapsule composition in soft tissue sarcomas. Clin Orthop Relat Res 2014;472:849-55.

22. Dede K, Salamon F, Landherr L, et al. Pathologic assessment of response to chemotherapy in colorectal cancer liver metastases after hepatic resection: which

Cite this article as: $\mathrm{Xi}$ W, Hou Y, Hu X, Xia Y, Jiang S, Wang H, Bai Q, Hou J, Guo J. Prognostic significance of pseudocapsule status in patients with metastatic renal cell carcinoma treated with tyrosine kinase inhibitors. Transl Androl Urol 2021;10(11):4132-4141. doi: 10.21037/tau-21-429 method to use? Pathol Oncol Res 2015;21:173-9.

23. Cheville JC, Lohse CM, Zincke H, et al. Sarcomatoid renal cell carcinoma: an examination of underlying histologic subtype and an analysis of associations with patient outcome. Am J Surg Pathol 2004;28:435-41.

24. Shuch B, Said J, La Rochelle JC, et al. Cytoreductive nephrectomy for kidney cancer with sarcomatoid histology--is up-front resection indicated and, if not, is it avoidable? J Urol 2009;182:2164-71.

25. Mian BM, Bhadkamkar N, Slaton JW, et al. Prognostic factors and survival of patients with sarcomatoid renal cell carcinoma. J Urol 2002;167:65-70.

26. Friedl P, Alexander S. Cancer invasion and the microenvironment: plasticity and reciprocity. Cell 2011;147:992-1009.

27. Ng IO, Lai EC, Fan ST, et al. Prognostic significance of pathologic features of hepatocellular carcinoma. A multivariate analysis of 278 patients. Cancer 1995;76:2443-8.

28. Lai EC, $\mathrm{Ng} \mathrm{IO}, \mathrm{Ng} \mathrm{MM}$, et al. Long-term results of resection for large hepatocellular carcinoma: a multivariate analysis of clinicopathological features. Hepatology 1990;11:815-8.

29. Motzer RJ, Bukowski RM, Figlin RA, et al. Prognostic nomogram for sunitinib in patients with metastatic renal cell carcinoma. Cancer 2008;113:1552-8.

30. Karacin C, Bilgetekin I, Basal FB, et al. Prognostic Importance of Metastatic Site in Intermediate-risk Group Metastatic Renal Cell Cancer Treated with Tyrosine Kinase Inhibitors. J Coll Physicians Surg Pak 2020;30:590-4.

31. Yao J, Hu X, Zhu Y, et al. Comparison between the efficacy of sunitinib and sorafenib in the first-line therapy on metastatic renal cell carcinoma. Chinese Journal of Clinical Medicine 2018;25:327-31. 


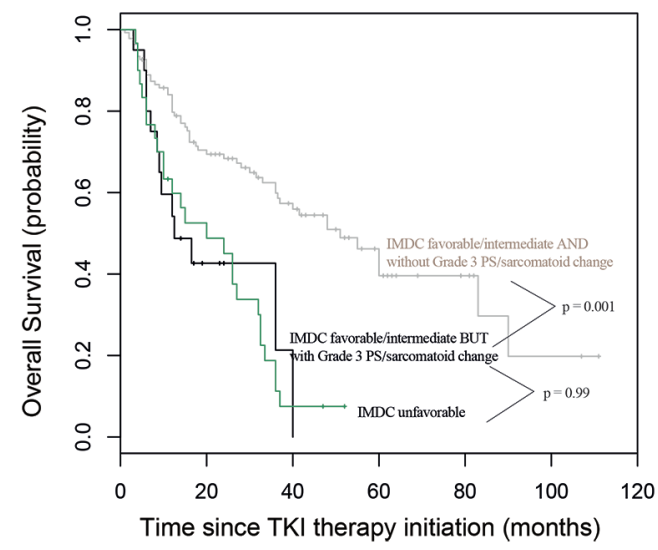

Figure S1 Overall survival according to IMDC risk group and PS absence and sarcomatoid change. Patients with intermediate risk according to IMDC criteria but without PS or had sarcomatoid change had much poorer overall survival, comparable to those with unfavorable risk. PS, pseudocapsule; IMDC, international Metastatic RCC Database Consortium. 

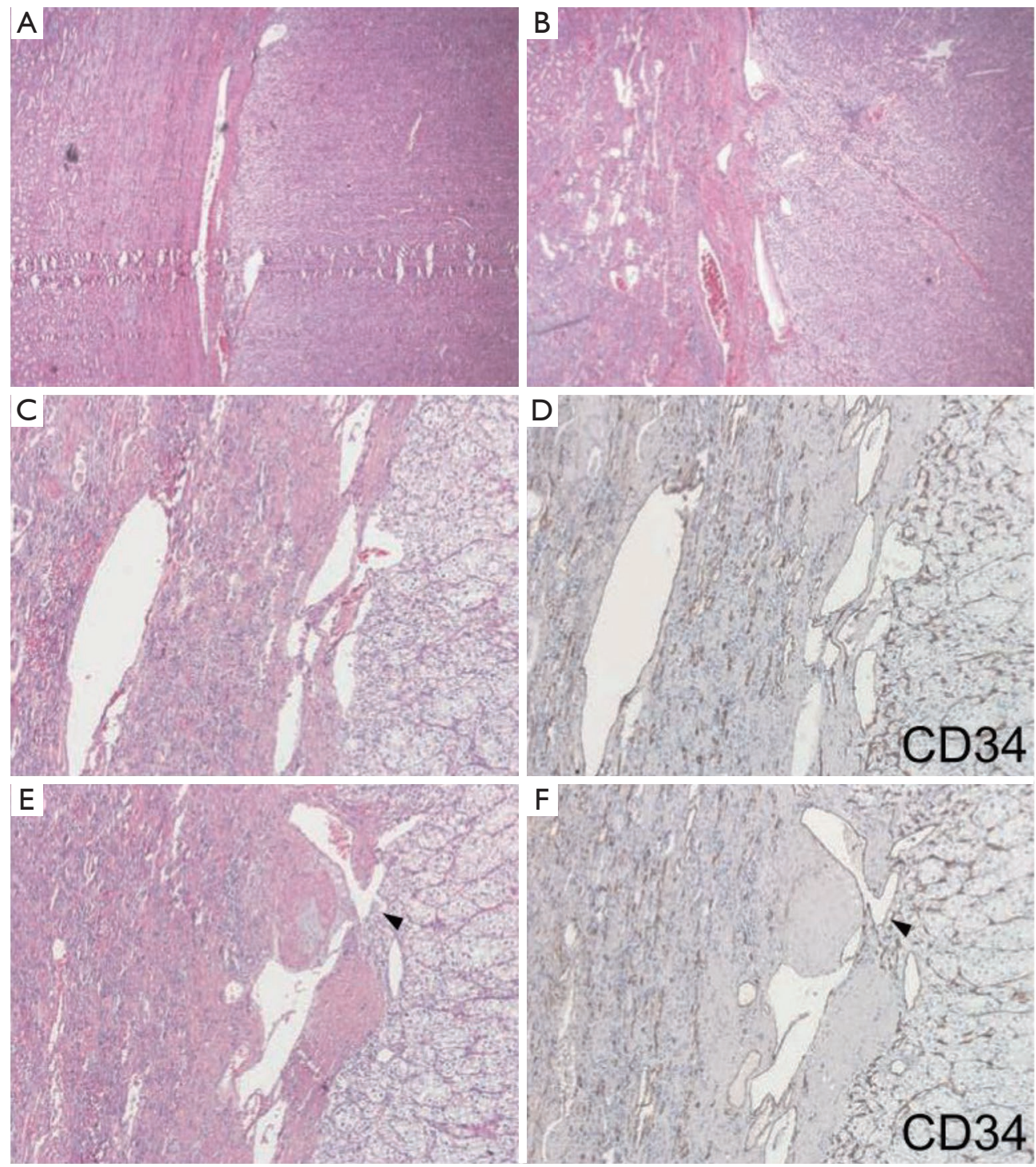

Figure S2 Representative pictures of vessels in PS. HE staining (A-C,E) and anti-CD34 staining (D,F) showed that PS contained more veins in large diameter (long diameter $>100 \mu \mathrm{m}$ ). In many situations, cancer cells at the edge were only separated by a thin layer of endotheliocytes to those large vessels (black arrowhead) (hematoxylin-eosin and immunohistochemistry, original magnification $\times 40$ ). PS, pseudocapsule; HE, hematoxylin-eosin. 


\section{HE}

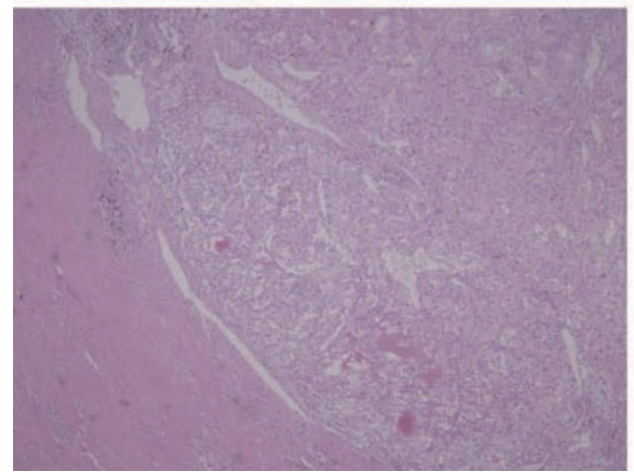

CD44 DAPI

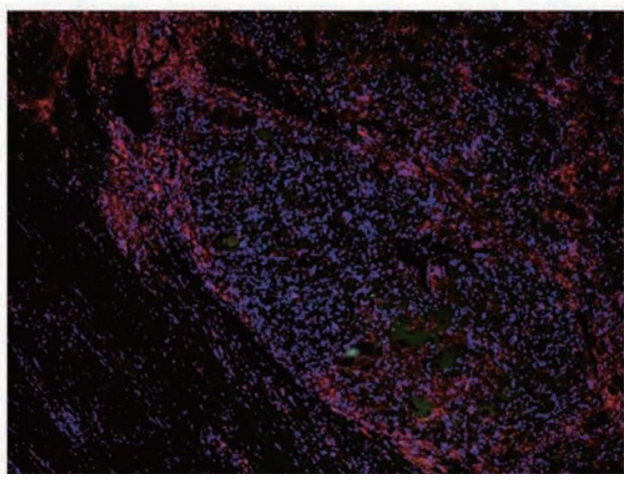

Figure S3 Representative pictures of $\mathrm{CD}_{4} 4^{+}$cancer cells surrounding PS. They mainly went alone the boundary between PS and tumor and were further enriched surrounding large vessels (hematoxylin-eosin and immunofluorescence, original magnification $\times 40$ ). PS, pseudocapsule.

Table S1 Relation between PS status and TKI response

\begin{tabular}{lcccc}
\hline TKI response & Grade 0 & Grade 1 & Grade 2 & Grade 3 \\
\hline PR & $3 / 19(15.8)$ & $19 / 61(30.0)$ & $18 / 96(19.6)$ & $1 / 12(8.3)$ \\
SD & $10 / 19(52.6)$ & $29 / 61(46.7)$ & $49 / 96(51.5)$ & $7 / 12(58.3)$ \\
PD & $6 / 19(31.6)$ & $13 / 61(23.3)$ & $29 / 96(28.9)$ & $4 / 12(33.3)$ \\
\hline
\end{tabular}

PS, pseudocapsule; TKI, tyrosine kinase inhibitors; PR, partial response; SD, stable disease; PD, progressive disease. 